\title{
THE STRUCTURE OF UMBRAL FLUXTUBES
}

\author{
D. DEGENHARDT and B. W. LITES \\ High Altitude Observatory, National Center for Atmospheric Research, \\ P.O. Box 3000, Boulder, CO 80307, U.S.A.
}

\begin{abstract}
Subsurface filamentation of sunspot magnetic fields has been postulated as a source of the visible small-scale structure of sunspot umbrae. We examine this possibility by investigating the magnetohydrodynamic structure of thin, vertical magnetized gas columns embedded in sunspot umbrae. These umbral fluxtubes are assumed to have weaker field strengths than the surrounding umbral atmosphere. The steady-state magnetohydrodynamic equations are solved numerically in the slender fluxtube approximation, thus allowing for stationary internal mass flows. We include the radiative exchange of heat between the umbral fluxtube and the ambient medium using a simple relaxation time approach.

The geometric shape of the steady flow solution is a gas column converging with height. We discuss the relationship of our results to observed properties of umbral brightenings (umbral dots). We show that, even if there is a large difference in magnetic field strength between the dot and the ambient medium in deeper layers, the field strengths are nearly equal in the observable layers, a result required by the observations. We also show that either high temperatures at the lower boundary of the dots or strong upflows are needed in order to produce bright continuum structures.
\end{abstract}

Key words: infrared: stars - MHD - Sun: magnetic fields - sunspots

\section{Introduction}

During the last decade the picture of an inhomogeneous subsurface structure of sunspots became popular. In this "cluster" model, the subsurface layers of the spot are fragmented into individual flux ropes which merge at a certain height to form a monolithic field structure at the surface (Parker 1979, Spruit 1981). This subsurface filamentation of the sunspot magnetic field would offer a natural explanation for the appearance of umbral dots, in the sense that umbral dots are caused by fieldfree plumes of gas pushing to the surface with high speed through a "magnetic valve" (Choudhuri 1986). However, contrary to prior theoretical treatment of this phenomenon, recent observations with very high spatial resolution indicate that the magnetic field strength is not greatly reduced within dots and they have only a very weak upflow, if any at all (Lites et al. 1991).

\section{Theoretical Model}

We change the Parker/Choudhuri configuration of umbral dots in a way that allows both for a (weaker) magnetic field and for a fluid flow in the regions they call "field-free" columns. Hence, we investigate the magnetohydrodynamic structure of thin, vertical magnetized gas columns ("umbral fluxtubes") embedded in a sunspot umbra, which is represented by model M of Maltby et al. (1986) permeated by a strong magnetic field.

We make the following assumptions: The basic geometry is such that the umbral fluxtubes are embedded in a cylinder of radius $r_{1}$ representing the ambient medium (see Fig. 1). At the lateral surface of this cylinder the external field is assumed to be vertical, that is the presence of the weak field tube is not felt beyond $r_{1}$. We 


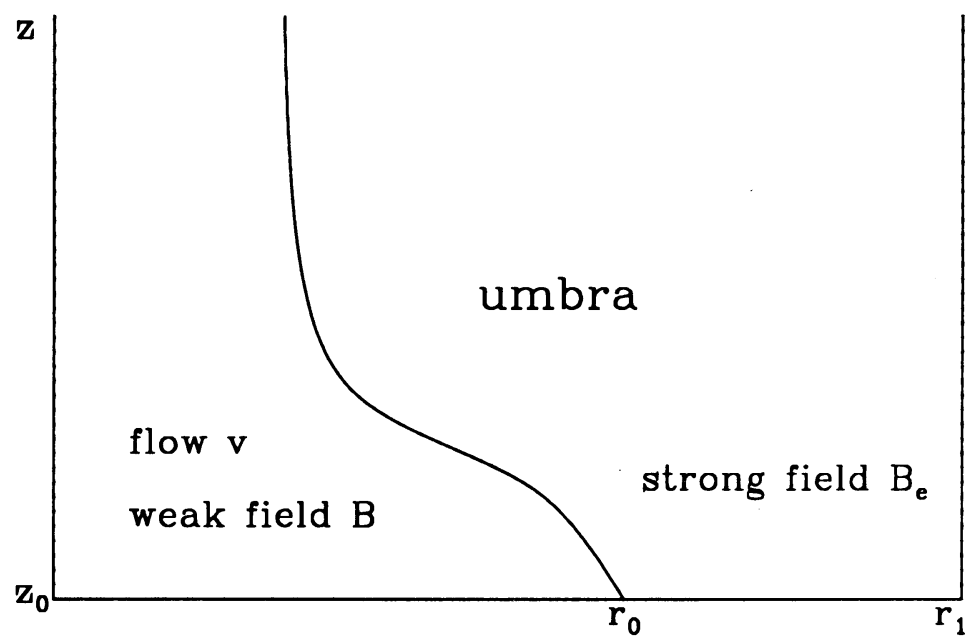

Fig. 1. Basic geometry of umbral fluxtubes. $r=0$ is the axis of a cylinder of radius $r_{1}$, where the umbral field is assumed to be vertical.

assume that the fluxtube (carrying the fluid flow) and the surroundings remain two distinct entities.

\subsection{Numerical Solution}

We adopt the steady-state one-dimensional magnetohydrodynamic equations of motion and of energy,

$$
\begin{aligned}
\rho v \frac{\mathrm{d} v}{\mathrm{~d} z} & =-\frac{\mathrm{d} p}{\mathrm{~d} z}-\rho g \\
v \frac{\mathrm{d} T}{\mathrm{~d} z} & =(1-\gamma) T\left(\frac{\chi_{\rho}}{\chi_{T}}\right) \Delta_{0}-\frac{1}{\rho c_{v}} \operatorname{div} \boldsymbol{F}_{R}
\end{aligned}
$$

which, together with the solenoidal condition for the magnetic field, the equation of continuity, and the ideal gas equation, are solved numerically using the slender fluxtube approximation (SFA) subject to the boundary condition

$$
p+\frac{B^{2}}{8 \pi}=p_{e}+\frac{B_{e}^{2}}{8 \pi}
$$

where the index " $e$ " refers to the external atmosphere. In eqs. (1)-(3), $\Delta_{0}$ is the zeroth order term of $\operatorname{div} v$ in the SFA, $\chi_{\rho}$ and $\chi_{T}$ describe the dependence of the mean molecular weight due to changes in ionization, $\boldsymbol{F}_{\boldsymbol{R}}$ denotes the radiative flux and all other symbols have their usual meanings. We include the radiative exchange of heat between the umbral fluxtube and the ambient medium using a simple relaxation time approach (for references see Degenhardt 1991). 

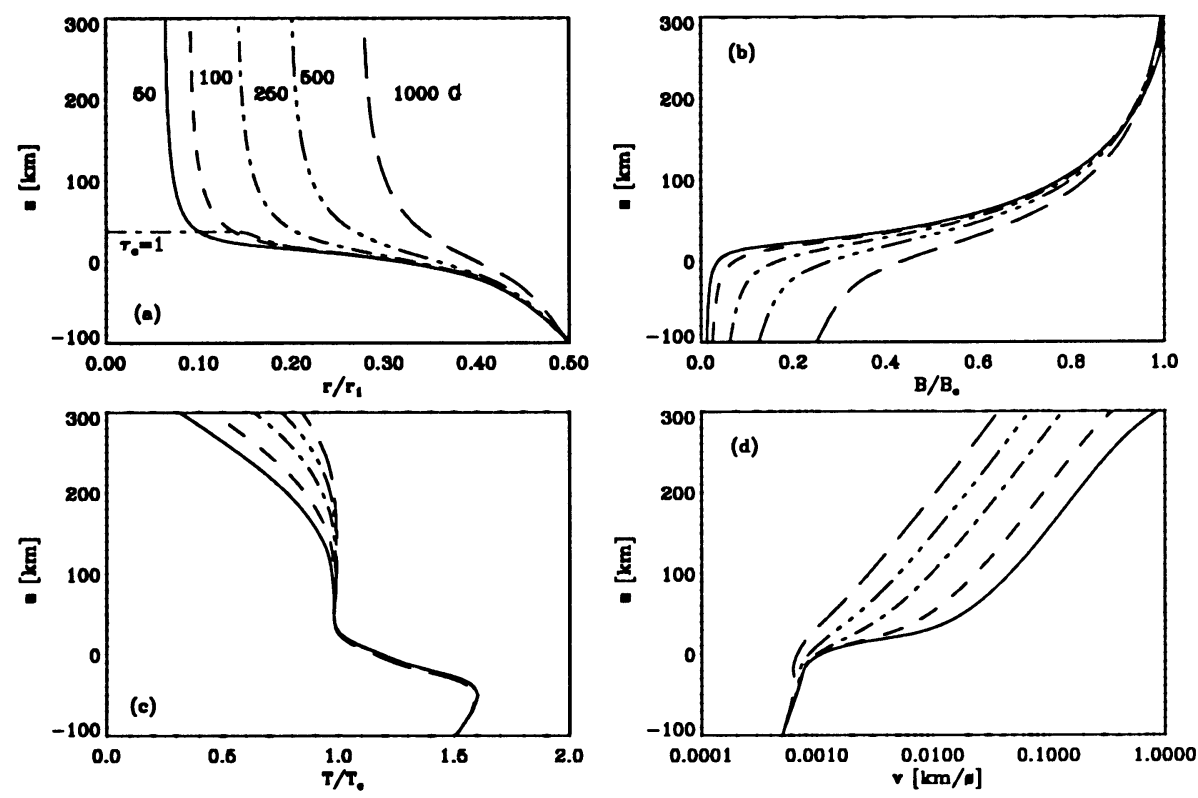

Fig. 2. (a) Magnetic field lines separating the umbral fluxtube and its surroundings, (b) field strength ratios, (c) temperature ratios and (d) flow speeds for varying ratios of magnetic field strengths at the lower boundary. The curves are labeled with the value of the internal magnetic field at the base.

The following model parameters can be prescribed at a certain height $z_{0}$ (see Fig. 1): the internal and external magnetic field strengths $B_{0}$ and $B_{e 0}$, the radius of the fluxtube $r_{0}$, the radius of the total cylinder $r_{1}$ in which the tube is embedded, the ratio of internal to external temperature $T_{0} / T_{e 0}$, and the velocity $v_{0}$.

Two different models are possible, corresponding to upflowing and downflowing gas, respectively. However, as there is no observational indication of a downflow in umbral dots, we only focus on models with upflowing material. The shape of the tube (and thus the external magnetic field) as well as the other physical quantities are calculated self-consistently during the computation.

\section{Results}

At first we investigate the influence of the ratio of magnetic field strengths at the lower boundary (see Fig. 2). We set the radius of the total cylinder to $r_{1}=1,000 \mathrm{~km}$ and $r_{0}=500 \mathrm{~km}$. The umbral field strength at the lower boundary is chosen to be $B_{e 0}=4,000 \mathrm{G}$ and we assume that the internal material is hotter than the umbra by a factor $T_{0} / T_{e 0}=1.5$. This is based upon the assumption that the convective energy transport in deeper layers is suppressed in the strong-field region relative to the umbral fluxtube. We impose a very weak upflow of $v_{0}=0.5 \mathrm{~m} \mathrm{~s}^{-1}$. Internal field strengths at the base are $B_{0}=50,100,250,500$ and $1,000 \mathrm{G}$, respectively, as indicated. 


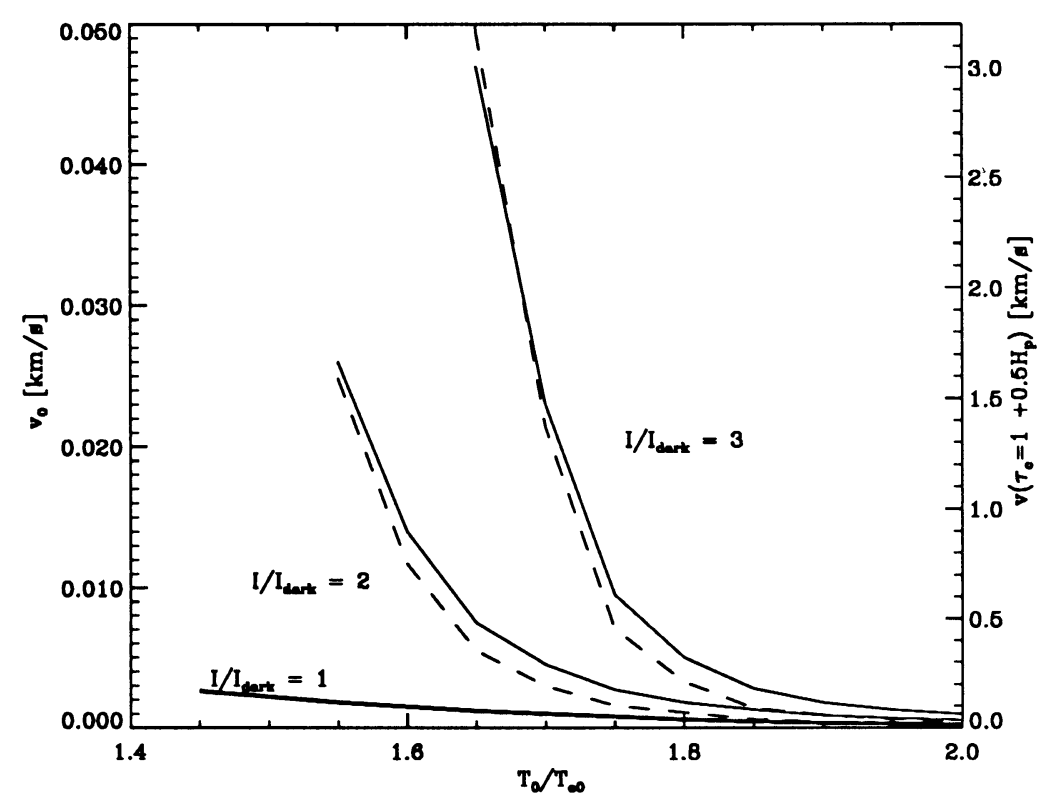

Fig. 3. Combinations of initial speed and temperature ratio which yield bright continuum structures, when observed from above, along the axis of the tube (see text).

The equilibrium structures are shown in the upper left panel of Figure 2. Due to the magnetic pressure of the umbral field the tubes with weaker field contract more strongly with height. The level $\tau_{c}=1$ is indicated for the model with $B_{0}=250 \mathrm{G}$. The upper right panel shows the variation of $B / B_{e}$ with height. As can be noted, the field strengths become nearly equal in the observable regions, even if the internal field at the base is very weak.

The lower right panel shows the flow speed along the tube. There is a stronger flow in the narrower tubes due to the Bernoulli effect. This strong flow yields a significant adiabatic cooling due to stratification in the upper portions of the tubes, which may be seen in the lower left panel, where the run of the temperature ratio $T / T_{e}$ is given.

Next, we examine what combinations of velocity $v_{0}$ and temperature ratio $T_{0} / T_{e 0}$ can produce bright structures in the continuum and may thus be identified with umbral dots. In principle, increasing the flow speed yields more heat advection into the upper portions of the tubes so that the hot, dense gas raises the $\tau_{c}=1$ level. The same effect can be achieved by increasing the temperature at the bottom of the tube.

We adopt an umbral field $B_{e 0}=3,300 \mathrm{G}$ and an internal field $B_{0}=150 \mathrm{G}$ at the lower boundary. With $r_{0}=500 \mathrm{~km}$ and $r_{1}=1,000 \mathrm{~km}$, these conditions result in a radius of $r \approx 125 \mathrm{~km}$ at $z=300 \mathrm{~km}$, a reasonable size for an umbral dot. At the same height the umbral field strength is $\sim 2,500 \mathrm{G}$. We carry out a simple LTE radiation diagnostic in the continuum for a hypothetical observation along the axis of the tube. The results are shown in Figure 3. 
The thick solid line indicates the combinations that result in structures as bright as the umbra itself. Choosing $v_{0}$ below this line leads to structures even darker than the umbra. The thinner solid lines give the combinations that result in structures twice or three times as bright as the umbra. We cannot simulate structures with $I / I_{\text {dark }}=2$ for $T_{0} / T_{e 0}<1.55$, because the very strong flow needed results in a shock, which cannot be treated by our code.

The dashed lines (corresponding to the label on the right axis) show the velocities at a level $1 / 2$ pressure scale height above the $\tau_{c}=1$ level. We feel that this is a rough approximation for the speeds which would be observed. As can be seen, one does not need to impose large flows to produce bright structures if the temperature enhancement at the bottom is sufficiently large.

\section{Conclusions}

Assuming that the umbrae of sunspots are divided into "normal" umbral regions and "umbral fluxtubes" with weaker field strengths, we find that, in the observable layers, the field strengths are approximately the same within and without, no matter how weak the tube field is below $\tau_{c}=1$ (in the umbra). Therefore, the best opportunity to observe reduced field strengths in umbral dots might be provided by the weak infrared FeI lines at the opacity minimum around $1.56 \mu \mathrm{m}$. Bruls et al. (1991) show that these lines form deeper in the umbral photosphere than the FeI lines in the visible spectrum commonly used as magnetic diagnostics.

In addition, we find that it is possible to construct umbral fluxtubes which appear as bright continuum structures (relative to the umbra) with upward flow speeds less than $0.5 \mathrm{~km} \mathrm{~s}^{-1}$, provided the temperature enhancement at the lower boundary is large enough.

The results of this preliminary investigation should be confirmed by simulations including a more realistic energy equation, especially involving a non-local energy transfer by radiation and by a more detailed radiation diagnostic, especially involving the infrared lines around $1.56 \mu \mathrm{m}$.

\section{Acknowledgements}

We are grateful to K. Ferriere for her instructive comments on this manuscript.

\section{References}

Bruls, J. H. M. J., Lites, B. W., Murphy, G. A.: 1991, in L. J. November (ed.), Solar Polarimetry, Proc. 11th National Solar Observatory/Sacramento Peak Summer Workshop, p. 444.

Choudhuri, A. R.: 1986, Astrophys. J. 302, 809.

Degenhardt, D.: 1991, Astron. Astrophys. 248, 637.

Lites, B. W., Bida, T. A., Johannesson, A., Scharmer, G. B.: 1991, Astrophys. J. 373, 683.

Maltby, P., Avrett, E. H., Carlsson, M., Kjeldseth-Moe, O., Kurucz, R. L., Loeser, R.: 1986, Astrophys. J. 306, 284.

Parker, E. N.: 1979, Astrophys. J. 230, 905.

Spruit, H. C.: 1981, in L. E. Cram and J. H. Thomas (eds.), The Physics of Sunspots, Sunspot, New Mexico, p. 98. 